\title{
RECURSIVE METHODS FOR COMPUTING \\ FINITE-TIME RUIN PROBABILITIES \\ FOR PHASE-DISTRIBUTED CLAIM SIZES
}

\author{
By D.A. Stanford AND K.J. Stroiński \\ Department of Statistical and Actuarial Sciences \\ University of Western Ontario, London, Ontario, Canada \\ N6A $5 B 7$
}

\begin{abstract}
Finite time ruin methods typically rely on diffusion approximations or discretization. We propose a new method by looking at the surplus process embedded at claim instants and develop a recursive scheme for calculating ruin probabilities. It is assumed that claim sizes follow a phase-type distribution. The proposed method is exact. The application of the method reveals where in the future the relative vulnerability to the company lies.
\end{abstract}

\section{KEYWORDS}

Finite-time ruin probability; Recursive methods; Phase-type distributions; Risk theory

\section{INTRODUCTION}

In this article we consider a classic model describing the evolution over time of the surplus of an insurance company. The value of the surplus process at time $t$ consists of the initial surplus plus premiums received, minus the value of claims that have occurred by time $t$. There is a vast literature describing the situation where the surplus process becomes negative for the first time.

The classic results concerning ruin probabilities were obtained by ARFWEDSON (1950), BEeKMAN (1966) and CramÉr (1955) and generalized by THORIN (1968). The classic problem was addressed also by PRABHU (1961 and 1965) and TAKács (1967). In the case of a compound Poisson claims process and a fixed rate of premium income $\mathrm{c}$ the non-ruin probability $\phi(a, t)$ over the finite horizon $t$ given an initial surplus $a$ is a solution of the integro-differential equation

$$
c \frac{\partial \phi(a, t)}{\partial a}=\frac{\partial \phi(a, t)}{\partial t}+\phi(a, t)-\int_{0}^{a} \phi(a-y, t) d B(y)
$$

where $B(\cdot)$ is the claim size distribution (see GERBER (1979)). 
The typical solution to this equation is stated in terms of Laplace transforms. Numerical difficulties involving the inversion of the Laplace transform solution were pointed out by JANSSEN and DELFOSSE (1982), TAYLOR (1978) and others. For the probability of eventual ruin, exact results were obtained for claim sizes given by combinations of exponential distributions and combinations of gamma distributions; see GERBER et al. (1987). Those results were generalized in DuFrESNE and GERBER (1988) for the family of combinations of shifted exponential distributions. Recursive calculations of ruin probabilities were developed by DEVYLDER and Goovaerts (1988) and SHIU (1988).

For more general cases, DICKSON and WATERS [(1991) and (1992)] present a discrete-time approximation for both the probability and the severity of ruin in finite time. A pertinent review of ruin theory results by TAYLOR (1985) contains references to many other texts on the subject.

It was recently shown by ASMUSSEN and ROLSKI (1991) that the exact results for the probability of eventual ruin can be obtained for distributions belonging to be so-called "phase-type" family. (A similar observation was also made by JANSSEN (1982).) In particular, if the claim size distribution is of the phase type, in the compound Poisson model, then the distribution of the maximal aggregate loss is a known phase-type distribution whose parameters are easily calculated. The probability of ruin is obtained as the tail of this distribution. Recently results involving phase-type distributions applied to the ruin problem were obtained by ASMUSSEN and BLADT (1992). However, most finite, continuous time ruin algorithms employ either diffusion approximations (GARRIDO (1988)) or discretize the surplus process, often employing PANJER's (1981) recursion formula.

The model we study can be described as follows: The initial surplus is $a$, and claims occur according to a Poisson process. Premiums are earned at a constant rate, and claim amounts are assumed to be non-negative, i.i.d. random variables with common distribution function $B(x)$. Claim amounts are assumed to be independent of the claim number process.

Since ruin occurs upon payment of claims, our approach is to observe the process embedded at claim instants only. The methods used lead to exact recursive formulae for the probability of ruin on a specific claim number. This in turn allows us to see where the relative vulnerability of the company lies over the duration of the process. We develop algorithms for the methods presented here assuming that the claim size distribution is of a phase-type.

Our interest in the probability of ruin on a specific claim has been motivated in part by the similarity between the surplus process and the workload process in a single-server queue. Similarities between ruin theory and queueing theory have been extensively explored in WILLMOT (1990).

In the next subsection necessary information about phase-type distributions is presented. In section 2 the general algorithm for phase-type distributions is described, and specific cases are considered. Numerical examples are presented in section 3 followed by conclusions in section 4 .

\subsection{Phase-type Distributions}

Phase-type distributions have become an extremely popular tool for applied probabilists wishing to generalize beyond the exponential while retaining some of 
its key properties. The phase-type family includes the exponential, mixture of exponentials, Erlangian and Coxian distributions as special cases. Among the appealing characteristics of phase-type distributions are the following closure properties : a) finite $n$-fold convolutions of phase-types are again of phase-type; b) a random modification (i.e. forward recurrence time) of a phase-type is again of phasetype; c) geometric mixtures of $n$-fold convolutions of phase-types are again of phase-type. This latest property is of particular interest vis-à-vis the ruin problem: couched in terms of its $M / G / 1$ waiting time analogue, NEUTs (1981) has shown that the distribution of the maximum aggregate loss is phase-type with easily determined parameters whenever the claim size distribution is phase-type as well. This fact was later used by JANSSEN (1982) and ASMUSSEN and ROLSKI (1991), who developed formulas for the ultimate probability of ruin for phase-type claim size distributions.

Phase-type distributions were first introduced by NeuTs in 1975, but the most popular standard reference for them has become NeUTS (1981). A shortened treatment can be stated as follows. Consider a Markov process with transient states $\{1,2, \ldots, m\}$ and absorbing state $(m+1)$, whose infinitesimal generator $Q$ has the form

$$
Q=\left[\begin{array}{ll}
T & t_{0} \\
0 & 0
\end{array}\right]
$$

The diagonal entries $T_{i i}$ are necessarily negative, other entries are non-negative, and $t_{0}=-T e$ represents the rates at which transitions occur from the individual transient states to the absorbing state.

Let the process start in state $i$ with probability $\alpha_{i}, i=1, \ldots, m+1$, and let $\boldsymbol{\alpha}=$ $\left(\alpha_{1}, \ldots, \alpha_{m}\right)$. (In many practical problems, $\alpha_{m+1}=0$.) Now let $B(x)$ denote the distribution of the time to absorption, $X$, into state $m+1$. The distribution $B(\cdot)$ thus described is said to be of "phase-type with representation $(\boldsymbol{\alpha}, T)$ ", and

$$
B(x)=1-\boldsymbol{\alpha} \exp (T x) \boldsymbol{e} ; x \geq 0 .
$$

Assuming $\alpha_{m+1}=0$ its density is $b(x)=\alpha \exp (T x) t_{0}, x \geq 0$, its Laplace-Stieltjes transform is $\Phi_{B}(s)=E\left\{e^{-s X}\right\}=\boldsymbol{\alpha}(s I-T)^{-1} \boldsymbol{t}_{\mathbf{0}}$, and its $n$th noncentral moment is given by

$$
\left.E\left\{X^{n}\right\}=n ! \alpha(-T)^{-n}\right) \boldsymbol{e}
$$

Many of these properties are quoted in the development which follows. The interested reader is directed to NEUTS (1981) for a rigorous treatment of phase-type distributions.

\section{Recursions for Phase-Distributed Claim Sizes}

The method we are about to describe works with the incomplete density for the reserve remaining after the $n$th claim occurs. (It is of course incomplete because ruin already have occurred.) Define

$$
p_{n}(y)=\frac{d}{d y} \operatorname{Pr}\{\text { non-ruin up to } n \text {th claim, and remaining reserve } \leqslant y\}
$$


and define the Laplace transforms

$$
\mathcal{L}_{n}(s)=\int_{0}^{x} e^{-s y} p_{n}(y) d y
$$

(Note in particular that $\mathcal{L}_{n}(0)=\operatorname{Pr}$ \{non-ruin up to $n$th claim $\}$.)

Next, define the "increment" between two consecutive claims as the difference between the revenue earned and the claim amount. Let $g(y)$ be its density (defined on $(-\infty, \infty)$ because the increment can assume both positive and negative values), and let

$$
G(s)=\int_{-\infty}^{\infty} e^{-s y} g(y) d y .
$$

Let the claim size have distribution function $B(y)$, density $b(y)$, and LaplaceStieltjes transform

$$
\Phi_{B}(s)=\int_{0}^{\infty} e^{-s y} d B(y)
$$

Since the number of claims is given by a Poisson process, inter-claim times (and hence revenue amounts earned between claims) are exponentially distributed. Furthermore, premiums are collected at a constant rate, so it follows that the revenue collected between consecutive claims is also exponentially distributed, and we denote the mean revenue by $(1 / \lambda)$. Since the increment is the difference between the revenue and the claim size, these definitions lead to the following results after straightforward manipulations :

$$
g(y)= \begin{cases}\Phi_{B}(\lambda) \lambda e^{-\lambda y} ; & y \geq 0 \\ \int_{0}^{\infty} \lambda e^{-\lambda t} b(t-y) d t ; & y<0\end{cases}
$$

Theorem : Let the claim size be given by a phase-type distribution with representation $(\alpha, T)$, and let $t_{0}=-T e$. Then

$$
\mathcal{L}_{n}(s)=\mathcal{L}_{n-1}(s) G(s)+\boldsymbol{v} \int_{x=0}^{\infty} p_{n-1}(x) \exp (T x) d x(s I+T)^{-1} \boldsymbol{t}_{0}
$$

where $v=\lambda \alpha(\lambda I-T)^{-1}$.

Proof: Having described the increment as above, the reserve after the $n$th claim is the sum of the reserve after the $(n-1)$ th claim and the ensuing increment. Therefore

$$
p_{n}(y)=\int_{0}^{\infty} p_{n-1}(x) g(y-x) d x
$$


The equivalent expression involving Laplace transforms is

$$
\mathcal{L}_{n}(s)=\int_{y=0}^{\infty} e^{-s y} p_{n}(y) d y=\int_{y=0}^{\infty} \int_{x=0}^{\infty} e^{-s y} p_{n-1}(x) g(y-x) d x d y .
$$

Due to absolute integrability, we can reverse the order of integration to obtain

$$
\begin{aligned}
\mathcal{L}_{n}(s) & =\int_{x=0}^{x} e^{-s x} p_{n-1}(x) \int_{y=0}^{\infty} e^{-s(y-x)} g(y-x) d y d x \\
& =\mathcal{L}_{n-1}(s) G(s)-\int_{x=0}^{x} e^{-s x} p_{n-1}(x) \int_{y=x}^{\infty} e^{s y} g(-y) d y d x
\end{aligned}
$$

Formula (2.8) applies for all non-negative generally distributed claim sizes. To complete the proof of the theorem, we must rely on properties of phase-type distributions. Recall that $b(y)=\alpha \exp (T y) t_{0}$. Therefore for $x<0$, we find

$$
\begin{aligned}
g(x) & =\int_{t=0}^{\infty} \lambda e^{-\lambda t} b(t-x) d t \\
& =\boldsymbol{\alpha} \int_{t=0}^{\infty} \lambda e^{-\lambda t} \exp (T(t-x)) d t_{0} \\
& =\lambda \boldsymbol{\alpha}(\lambda I-T)^{-1} \exp (-T x) t_{0}=v \exp (-T x) \boldsymbol{t}_{0}
\end{aligned}
$$

Consequently we can evaluate the inner integral of the 2 nd term of (2.8) as follows :

$$
\begin{aligned}
\int_{y=x}^{\infty} e^{s y} g(-y) d y & =v \int_{y=x}^{\infty} e^{s y} \exp (T y) d y t_{0} \\
& =v \int_{y=x}^{\infty} \exp ((s I+T) y) d y t_{0} \\
& =-e^{s x} v \exp (T x)(s I+T)^{-1} t_{0}
\end{aligned}
$$

When (2.9) is substituted into (2.8), equation (2.6) is obtained.

Remark: By evaluating (2.6) at $s=0$, one finds

$\operatorname{Pr}\{$ non-ruin up to the $n$th claim $\}=$

$\operatorname{Pr}\{$ non-ruin up to the $(n-1)$ th claim $\}$

$$
+v \int_{x=0}^{\infty} p_{n-1}(x) \exp (T x) d x T^{-1} t_{0}
$$


from which one concludes that

$$
P(n) \equiv \operatorname{Pr}\{\text { ruin on the } n \text {th claim }\}=v \int_{x=0}^{x} p_{n-1}(x) \exp (T x) d x e .
$$

In what follows, we develop computational algorithms from (2.6) and (2.10) for the specific cases of i) exponential, ii) mixtures of exponential claim size distributions, and iii) Erlang- $N$ distributions.

\subsection{Recursive Algorithm for Exponential Claim Sizes}

If one assumes that claim sizes are exponentially distributed with mean $(1 / \mu)$, we can simplify the recursions for $\mathcal{L}_{n}(s)$ and $P(n)$ greatly. All of the matrix quantities reduce to scalar results. In particular:

$$
\begin{gathered}
G(s)=(\lambda / \lambda+s)(\mu / \mu-s), T=\mid-\mu], t_{0}=\mu, \\
v=\lambda /(\lambda+\mu), \\
(s I+T)^{-1}=1 /(s-\mu), \text { and } \exp (T x)=e^{-\mu x} .
\end{gathered}
$$

Therefore (2.6) becomes

$$
\mathcal{L}_{n}(s)=(\mu / \mu-s)\left[(\lambda / \lambda+s) \mathcal{L}_{n-1}(s)-(\lambda / \lambda+\mu) \mathcal{L}_{n-1}(\mu)\right]
$$

and

$$
P(n)=(\lambda / \lambda+\mu) \mathcal{L}_{n-1}(\mu) .
$$

For the remainder of this section, let $\Phi \equiv \Phi_{B}(\lambda)=(\mu / \mu+\lambda)$. We seek an algorithm to determine the form of $\mathcal{L}_{n}(s)$ in terms of $\mathcal{L}_{n-1}(s)$, and the corresponding expression for $P(n)$. If the initial reserve is $a$, then $\mathcal{L}_{0}(s)=e^{-a s}$. Thus from (2.12)

$$
P(1)=(1-\Phi) L_{0}(\mu)=(1-\Phi) e^{-a \mu} .
$$

Using (2.11) we can find $\mathcal{L}_{1}(s)$ as follows

$$
\begin{aligned}
\mathcal{L}_{1}(s) & \left.=\lambda \mu /(\mu-s) \mid e^{-a s} /(\lambda+s)-e^{-a \mu} /(\lambda+\mu)\right] \\
& =\lambda \mu e^{-a \mu}\left[(\lambda+\mu) e^{a(\mu-s)}-(\lambda+s)\right] /\{(\mu-s)(\lambda+s)(\lambda+\mu)\} \\
& =\lambda \mu e^{-a \mu}\left[(\mu-s)+(\lambda+\mu) \sum_{k=1}^{\infty}(a(\mu-s))^{k} / k !\right] /[(\mu-s)(\lambda+s)(\lambda+\mu)] \\
& =\lambda /(\lambda+s) e^{-a \mu}\left[\Phi+(a \mu) \sum_{k=1}^{\infty}(a(\mu-s))^{k-1} / k !\right] .
\end{aligned}
$$

Again using (2.12) for $n=2$ we find

$$
P(2)=(1-\Phi) \mathcal{L}_{1}(\mu)=(1-\Phi)^{2} e^{-a \mu}[\Phi+a \mu]
$$


and (2.11) gives rise to the following expression:

$$
\begin{aligned}
L_{2}(s)= & e^{-a \mu}\left[(\Phi+a \mu)\left\{\Phi(\lambda / \lambda+s)^{2}+\Phi(1-\Phi)(\lambda / \lambda+s)\right\}\right. \\
& \left.+(a \mu)^{2}(\lambda / \lambda+s)^{2} \sum_{k=2}^{\infty}(a(\mu-s))^{k-2} / k !\right]
\end{aligned}
$$

We now establish the general form of $\mathcal{L}_{n}(s)$.

Theorem: For $n \geq 1$, the Laplace transform of the distribution of the reserve following the $n$th claim is given by

$$
L_{n}(s)=e^{-u \mu}\left[\sum_{j=1}^{n} c_{j}^{(n)}(\lambda / \lambda+s)^{j}(1-\Phi)^{n-j}+(a \mu)^{n}(\lambda / \lambda+s)^{n} \sum_{k=n}^{\infty} \frac{(a(\mu-s))^{k-n}}{k !}\right]
$$

Furthermore, the coefficients $c_{j}^{(n)}$ at the $n$th stage are related to those at the $(n-1)$ th stage via

$$
c_{j}^{(n)}=\Phi\left\{\sum_{k=\max (1 . j-1)}^{n-1} c_{k}^{(n-1)}+\frac{(a \mu)^{n-1}}{(n-1) !}\right\} ; j=1, \ldots, n
$$

Proof: The proof proceeds via induction. For $n=1,(2.14)$ show that the form of (2.17) is correct, with $c_{1}^{(1)}=\Phi$. For $n=2,(2.16)$ reveals that $c_{1}^{(2)}=c_{2}^{(2)}=\Phi^{2}+(a \mu) \Phi$.

Now assume that (2.17) is valid up to index $n=N-1$. Thus

$$
\begin{aligned}
\mathcal{L}_{N-1}(s)= & e^{-a \mu}\left[\sum_{j=1}^{N-1} c_{j}^{(N-1)}(\lambda / \lambda+s)^{j}(1-\Phi)^{N-1-j}\right. \\
& \left.+(a \mu)^{N-1}(\lambda / \lambda+s)^{N-1} \sum_{k=N-1}^{\infty} \frac{(a(\mu-s))^{k-N+1}}{k !}\right]
\end{aligned}
$$

Substituting (2.19) into (2.11) for $n=N$ we find

$$
\begin{aligned}
\mathcal{L}_{N}(s)= & \mu /(\mu-s) e^{-a \mu}\left[\sum_{j=1}^{N-1} c_{j}^{(N-1)}(1-\Phi)^{N-1-j}\left((\lambda / \lambda+s)^{j+1}-(\lambda / \lambda+\mu)^{j+1}\right)\right. \\
& \left.+(a \mu)^{N-1}\left\{\frac{(\lambda / \lambda+s)^{N}}{(N-1) !}-\frac{(\lambda / \lambda+\mu)^{N}}{(N-1) !}\right\}+(\lambda / \lambda+s)^{N} \sum_{k=N}^{\infty} \frac{(a(\mu-s))^{k-N+1}}{k !}\right] \\
= & e^{-a \mu\left[\sum_{j=1}^{N-1} c_{j}^{(N-1)}(1-\Phi)^{N-1-j}\left(\frac{\mu}{\lambda+\mu}\right)\left(\frac{\lambda}{\lambda+s}\right) \sum_{l=0}^{j}(\lambda / \lambda+s)^{l}(\lambda / \lambda+\mu)^{j-l}\right.} \\
& +\frac{(a \mu)^{N-1}}{(N-1) !}\left(\frac{\mu}{\lambda+\mu}\right)\left(\frac{\lambda}{\lambda+s}\right) \sum_{l=0}^{N-1}(\lambda / \lambda+s)^{l}(\lambda / \lambda+\mu)^{N-1-1} \\
& \left.+(a \mu)^{N}(\lambda / \lambda+s)^{N} \sum_{k=N}^{\infty} \frac{(a(\mu-s))^{k-N}}{k !}\right]
\end{aligned}
$$


The infinite sum in (2.20) already satisfies the form required by (2.17) for $n=N$. It remains for us to rearrange the other terms in (2.20) and establish the recursion posed by (2.18). Note that these can be written as

$$
\begin{array}{r}
e^{-a \mu}\left[\sum_{l=0}^{N-1} \Phi \sum_{j=\max (1, l)}^{N-1} c_{j}^{(N-1)}(1-\Phi)^{N-1-j}(\lambda / \lambda+s)^{l+1}(\lambda / \lambda+\mu)^{j-l}\right. \\
\left.+\sum_{l=0}^{N-1} \Phi \frac{(a \mu)^{N-1}}{(N-1) !}(\lambda / \lambda+s)^{l+1}(\lambda / \lambda+\mu)^{N-1-l}\right] \\
=e^{-a \mu}\left[\sum_{l=1}^{N}(\lambda / \lambda+s)^{l}(1-\Phi)^{N-l} \Phi\left\{\sum_{k=\max (1, l-1)}^{N-1} c_{k}^{(N-1)}+\frac{(a \mu)^{N-1}}{(N-1) !}\right\}\right]
\end{array}
$$

A comparison of equivalent powers of $(\lambda / \lambda+s)$ between $(2.21)$ and the first sum of (2.17) establishes (2.18) and completes the proof by induction.

When (2.17) is substituted into (2.12) one obtains

$$
P(n)=(1-\Phi)^{n} e^{-a \mu}\left\{\sum_{j=1}^{n-1} c_{j}^{(n-1)}+\frac{(a \mu)^{n-1}}{(n-1) !}\right\} .
$$

However, in light of (2.18) this can be restated as follows.

\section{Corollary :}

$$
P(n)=(1-\Phi)^{n} e^{-a \mu} c_{1}^{(n)} / \Phi, \quad n=1,2, \ldots
$$

The algorithm for determining $P(n)$ thus consists of (2.18) and (2.22), starting from $c_{1}^{(1)}=\Phi$.

\subsection{Recursive Algorithm for Mixtures of Exponentials}

Mixtures of a finite number of exponential distributions have been used frequently as a generalization of the single-exponential case, see for instance GERBER (1979).

The phase-type formulation of a mixture of $K$ exponentials has the following form : $\boldsymbol{\alpha}=\left[p_{1}, p_{2}, \ldots, p_{K}\right] ; T=-\operatorname{diag}\left[\mu_{1}, \mu_{2}, \ldots, \mu_{K}\right]$, and $\boldsymbol{t}_{0}=\left[\mu_{1}, \mu_{2}, \ldots, \mu_{K}\right]^{\prime}$. Similarly $\Phi_{B}(s)$

$=\left[p_{1}, p_{2}, \ldots, p_{K}\right] \operatorname{diag}\left[\left(\mathrm{s}+\mu_{1}\right)^{-1},\left(s+\mu_{2}\right)^{-1}, \ldots,\left(s+\mu_{K}\right)^{-1}\right]\left[\mu_{1}, \mu_{2}, \ldots, \mu_{K}\right]^{\prime}$

$=\sum_{i=1}^{K} p_{i} \mu_{i} /\left(s+\mu_{i}\right)$

The other computations for (2.6) are equally straightforward, and the resulting recursion for $\mathcal{L}_{n}(s)$ is

$$
\mathcal{L}_{n}(s)=\sum_{i=1}^{K} p_{i}\left(\mu_{i} / \mu_{i}-s\right)\left[\mathcal{L}_{n-1}(s)(\lambda / \lambda+s)-\mathcal{L}_{n-1}\left(\mu_{i}\right)\left(\lambda / \lambda+\mu_{i}\right)\right]
$$


and the probability of ruin on the $n$th claim is

$$
P(n)=\sum_{i=1}^{K} p_{i}\left(\lambda / \lambda+\mu_{i}\right) \mathcal{L}_{n-1}\left(\mu_{i}\right)
$$

Again, we seek explicit expressions for $\mathcal{L}_{n}(s)$ and $P(n)$ which can be calculated recursively. Defining $\Phi_{i}=\left(\mu_{i} / \lambda+\mu_{i}\right)$, we find (since $\mathcal{L}_{0}(s)=e^{-a s}$ ):

$$
\begin{gathered}
P(1)=\sum_{i=1}^{K} p_{i}\left(1-\Phi_{i}\right) e^{-a \mu_{i}} \\
\mathcal{L}_{0}(s)=(\lambda / \lambda+s) \sum_{i=1}^{K} p_{i} e^{-a \mu_{i}}\left[\Phi_{i}+\left(a \mu_{i}\right) \sum_{k=1}^{\infty} \frac{\left(a\left(\mu_{i}-s\right)\right)^{k-1}}{k !}\right]
\end{gathered}
$$

from which $P(2)$ can be readily calculated using (2.24). Determination of $\mathcal{L}_{n}(s)$ for $n \geq 2$ in the general case becomes increasingly complicated due to the need to evaluate $\mathcal{L}_{n-1}\left(\mu_{i}\right)$ at all $K$ rates. Tractable results are, however, available for two important sub-cases. For both (2.24) can then be used to find the corresponding probabilities.

Case A : No Initial Reserve $(a=0)$ : In this case

$$
\mathcal{L}_{1}(s)=(\lambda / \lambda+s) \sum_{i=1}^{K} p_{i} \Phi_{i}
$$

and similar methods to those of the previous section can be used to establish the following recursion:

$$
\mathcal{L}_{n}(s)=\sum_{j=1}^{n} c_{j}^{(n)}(\lambda / \lambda+s)^{j} \quad n=1,2, \ldots
$$

where

$$
c_{j}^{(n)}=\sum_{k=\max (1, j-1)}^{(n-1)} c_{k}^{(n-1)}\left\{\sum_{i=1}^{K} p_{\mathrm{i}} \Phi_{i}\left(1-\Phi_{i}\right)^{k+1-j}\right\} \quad j=1,2, \ldots, n
$$

and where

$$
c_{1}^{(1)}=\sum_{i=1}^{K} p_{i} \Phi_{i}
$$

\section{Case B : Mixture of 2 Exponentials}

Theorem: For the case of a mixture of 2 exponentials the following relationship applies for $\mathcal{L}_{n}(s), n \geq 1$ :

$$
\mathcal{L}_{n}(s)=\sum_{j=1}^{n} c_{j}^{(n)}(\lambda / \lambda+s)^{j}+(\lambda / \lambda+s)^{n} A^{(n)}(s)
$$


where

$$
\begin{aligned}
A^{(n)}(s)= & \sum_{k=n}^{\infty} \frac{a^{k-n}}{k !} \sum_{m=1}^{2} p_{m}\left\{D_{m}^{(n)}\left(\mu_{m}-s\right)^{k-n}\right. \\
& \left.+\sum_{j=0}^{n-2} f_{j m}^{(n)} \sum_{l=0}^{k-n}\left(\begin{array}{c}
k-n-l+j \\
j
\end{array}\right)\left(\mu_{m}-s\right)^{l}\left(\mu_{m}-\mu_{3-m}\right)^{k-n-l}\right\},
\end{aligned}
$$

where the coefficients $c_{j}^{(n)}, D_{m}^{(n)}$ and $f_{j m}^{(n)}, n \geq 2$, satisfy the recursions

$$
\begin{gathered}
c_{j}^{(n)}=\sum_{i=1}^{2} p_{i} \Phi_{i}\left[\sum_{k=\max (1, j-1)}^{n-1} c_{k}^{(n-1)}\left(\lambda / \lambda+\mu_{i}\right)^{k+1-j}+\left(\lambda / \lambda+\mu_{i}\right)^{n-j} A_{i}^{(n-1)}\right], \\
f_{j m}^{(n)}= \begin{cases}r_{m} f_{0 m}^{(n-1)}+r_{3-m} D_{m}^{(n-1)} & j=0 \\
r_{m} f_{j m}^{(n-1)}+r_{3-m} f_{j-1, m}^{(n-1)} & j=1,2, \ldots, n-3 \\
r_{3-m} f_{n-3, m}^{(n-1)} & j=n-2\end{cases}
\end{gathered}
$$

where $r_{m}=p_{m} a \mu_{m}$ and

$$
A_{i}^{(n-1)} \equiv A^{(n-1)}\left(\mu_{i}\right) .
$$

The coefficients are initialized as follows:

$$
c_{1}^{(1)}=\sum_{i=1}^{2} p_{i} e^{-a \mu_{i}} \Phi_{i} ; \quad D_{i}^{(1)}=e^{-a \mu_{i}}\left(a \mu_{i}\right), \quad i=1,2
$$

and

$$
f_{0 m}^{(2)}=r_{3-m} D_{m}^{(1)} ; \quad m=1,2
$$

Proof: Follows after tedious but straightforward substitution using (2.23) and (2.26).

\subsection{Recursive Algorithm for Erlang- $N$ Claims}

The Erlang- $N$ distribution is the $N$-fold convolution of the exponential distribution, and thus forms a subset of the family of Gamma distributions. It is often used to model distributions which are less variable relative to the mean than the exponential. Let the mean per exponential stage be $1 / \theta$. The Erlang- $N$ can be viewed within the phase-type framework as a process successively moving through states 1 through $m$ prior to absorption into state $m+1$. Thus $\boldsymbol{\alpha}=[1,0, \ldots, 0]$;

$$
T=\left[\begin{array}{cccc}
-\theta & \theta & & 0 \\
& -\theta & \theta & \\
0 & & \ddots & -\theta
\end{array}\right] \quad ; \text { and } t_{0}=\left[\begin{array}{c}
0 \\
\vdots \\
0 \\
\theta
\end{array}\right]
$$


Theorem : The recursion for $\mathcal{L}_{n}(s)$ when the claim size distribution is Erlang- $N$ as described above is as follows:

$$
\begin{aligned}
& \mathcal{L}_{n}(s)=\left\{\left[\mathcal{L}_{n-1}(s)(\lambda / \lambda+s), 0, \ldots, 0\right]\right. \\
& \left.-\left[p \mathcal{L}_{n-1}(\theta), p(-\theta) \mathcal{L}_{n-1}^{\prime}(\theta)+p q \mathcal{L}_{n-1}(\theta), \ldots, \sum_{l=0}^{N-1} p q^{l} \frac{(-\theta)^{N-1-l}}{(N-1-l) !} \mathcal{L}_{n-1}^{(N-1-l)}(\theta)\right]\right\} \\
& \times\left[\begin{array}{c}
(\theta / \theta-s)^{N} \\
(\theta / \theta-s)^{N-1} \\
\vdots \\
(\theta / \theta-s)
\end{array}\right]
\end{aligned}
$$

where $p=(\lambda / \lambda+\theta)$ and $q=1-p$.

Proof : One can show after elementary calculations that

$$
\begin{gathered}
(-s I-T)^{-1} \boldsymbol{t}_{\mathbf{0}}=\left[(\theta / \theta-s)^{N},(\theta / \theta-s)^{N-1}, \ldots,(\theta / \theta-s)\right]^{\prime} \\
\left.v=\lambda \alpha(\lambda I-T)^{-1}=[\lambda / \lambda+\theta), \lambda \theta /(\lambda+\theta)^{2}, \ldots, \lambda \theta^{N-1} /(\lambda+\theta)^{N}\right]
\end{gathered}
$$

and

$$
\exp (T x)=\left[\begin{array}{ccccc}
f_{0}(\theta x) & f_{1}(\theta x) & f_{2}(\theta x) & \ldots & f_{N-1}(\theta x) \\
0 & f_{0}(\theta x) & f_{1}(\theta x) & \ldots & f_{N-2}(\theta x) \\
0 & 0 & f_{0}(\theta x) & \ldots & f_{N-3}(\theta x) \\
\vdots & \vdots & \vdots & & \\
0 & 0 & 0 & & f_{0}(\theta x)
\end{array}\right]
$$

where $f_{k}(\theta x)=(-1)^{k} \sum_{l=k}^{\infty} \frac{(-\theta x)^{l}}{k !(l-k) !}=\frac{(\theta x)^{k}}{k !} e^{-\theta x}$. Therefore

$$
\begin{aligned}
& \int_{x=0}^{\infty} p_{n-1}(x) \exp (T x) d x \\
& =\left[\begin{array}{cccc}
\mathcal{L}_{n-1}(\theta) & (-\theta) \mathcal{L}_{n-1}^{\prime}(\theta) & \theta^{2} \mathcal{L}_{n-1}^{\prime \prime}(\theta) / 2 & (-\theta)^{N-1} \mathcal{L}_{n-1}^{(N-1)}(\theta) /(N-1) ! \\
0 & \mathcal{L}_{n-1}(\theta) & (-\theta) \mathcal{L}_{n-1}^{\prime}(\theta) & (-\theta)^{N-2} \mathcal{L}_{n-1}^{(N-2)}(\theta) /(N-2) ! \\
0 & 0 & \mathcal{L}_{n-1}(\theta) & (-\theta)^{N-3} \mathcal{L}_{n-1}^{(N-3)}(\theta) /(N-3) ! \\
\vdots & \vdots & \vdots & \vdots \\
0 & 0 & 0 & \mathcal{L}_{n-1}(\theta)
\end{array}\right] .
\end{aligned}
$$


Substitution of these expressions into (2.6) in light of (2.5) gives rise to the theorem's result. Substitution of $s=0$ in (2.34) leads to the following result after elementary manipulations.

Corollary : In the case of Erlang- $N$ claim-size distributions, the probability of ruin on the $n$th claim is given by

$$
P(n)=\sum_{i=0}^{N-1} \mathcal{L}_{n-1}^{(i)}(\theta)\left\{(-\theta)^{i}\left[1-q^{N-i}\right] / i !\right\} .
$$

In the case of Erlang-2 claims, this reduces to

$$
P(n)=\mathcal{L}_{n-1}(\theta)\left(1-q^{2}\right)-p \theta \mathcal{L}_{n-1}^{\prime}(\theta) .
$$

Using the same methods as before, one can again develop recursions for $\mathcal{L}_{n}(s)$. We state here as an example the recursion for the case of Erlang- 2 claim sizes:

$$
\mathcal{L}_{n}(s)=e^{-a \theta}\left[\sum_{j=1}^{n} c_{j}^{(n)}(\lambda / \lambda+s)^{j}+(a \theta)^{2 n}(\lambda / \lambda+s)^{n} \sum_{k=2 n}^{\infty} \frac{(a(\theta-s))^{k-2 n}}{k !}\right]
$$

where the coefficients $c_{j}^{(n)}$ satisfy the recursion:

$$
\begin{aligned}
c_{j}^{(n)}= & {\left[\frac{(a \theta)^{2 n-2} q^{2}}{(2 n-2) !}(n+1-j)+\frac{(a \theta)^{2 n-1}}{(2 n-1) !} q\right] p^{n-j} } \\
& +q^{2} \sum_{k=\max (2, j)}^{n} c_{k-1}^{(n-1)} p^{k-j}(k+1-j) . \quad j=1, \ldots, n ; n=1,2, \ldots
\end{aligned}
$$

The recursion starts with $c_{1}^{(1)}=q^{2}+a \theta q$.

\section{NUMERICAL EXAMPLES}

A series of numerical examples have been carried out to demonstrate the effect of the security loading, the initial reserve, and the claim size distribution on the probability of ruin. The results are displayed in Figures 1 through 10 .

Figures 1 and 2 display the cumulative probability of ruin for exponentially distributed claims. In Figure 1, it is assumed that there is no initial reserve. Although the probabilities of this and all later graphs are in fact valid only for integer claim numbers, continuous trajectories have been fitted to these points to show the overall trend. Figure 1 shows that these trajectories approach their ultimate limit of $1 /(1+\theta)$ (where $\theta$ is the relative security loading). This limit is a well-known result in risk theory; see for example Bowers et al. (1986) p. 359, eq. (12.5.2). The speed of convergence to this limit increases with $\theta$. This is not surprising-if the security loading is large, one would expect either to be ruined very soon, or else to have built up enough surplus to weather further fluctuations. For small $\theta$, a smaller reserve accumulates, so the period of vulnerability lasts longer. 


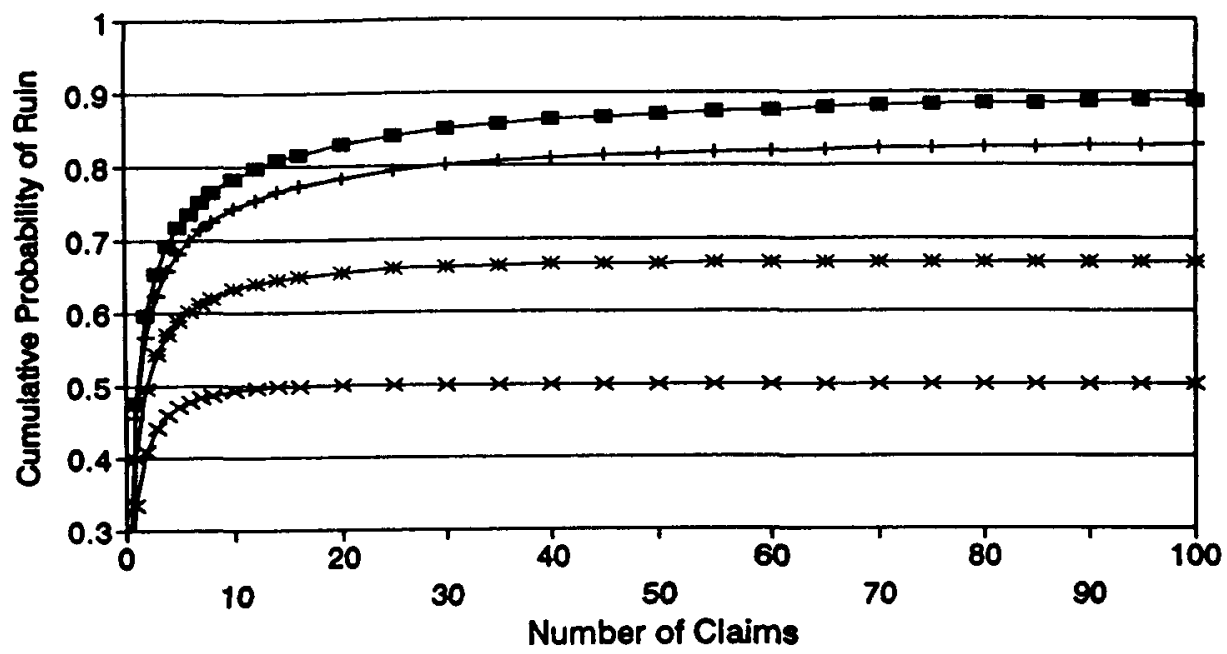

\section{$\rightarrow-$ theta $=10 \% \rightarrow$ theta $=20 \% \rightarrow$ theta $=50 \% \rightarrow$ theta $=100 \%$}

FigurE 1. Effect of security loading (no initial reserve).

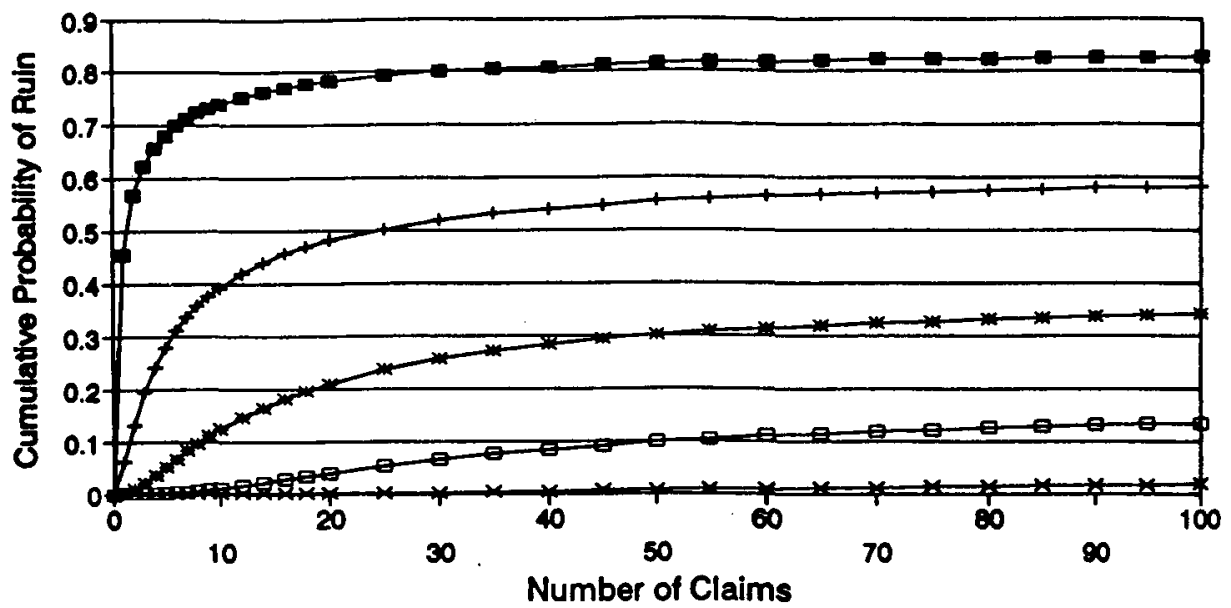

$$
\begin{aligned}
& \rightarrow-I R R=0 \rightarrow I R R=2 \rightarrow-I R R=5 \\
& \square I R R=10 \rightarrow-I R R=20
\end{aligned}
$$

FIGURE 2. Effect of initial reserve (security loading $=20 \%$ ). 
Figure 2 displays the cumulative probability of ruin as a function of the time and the initial reserve level. Here the acronym IRR refers to the initial reserve ratio, defined are the initial reserve divided by the expected amount of a single claim. The security loading is $20 \%$. The figure shows that even small reserves have a major benefit as opposed to having no reserve at all. For larger reserves, the commencement of the period of vulnerability is delayed due to the extremely small chance of a large number of claims during a single time unit.

The probability of ruin on a given claim number reveals the relative vulnerability of the company. This vulnerability is explored in Figures 3 though 6, where exponential claim sizes have been assumed. Figures 3 and 4 show that with a non-zero initial surplus, the ruin probabilities for the process with a smaller relative security loading $\theta$ are spread over a much wider range than those for larger $\theta$.

Figures 5 and 6 show that for larger initial surpluses, the ruin probabilities are again spread over a wider range for smaller $\theta$ 's. Further, the larger initial surplus reduces the possibility of early ruin, however, the relative vulnerability is more spread out.

To demonstrate the effect of more variable claim size distributions, Figures 7 and 8 display the probabilities of ruin on the $n$th claim for a series of mixtures of two exponentials with balanced means. This balanced means assumption $\left(p_{1} / \mu_{1}=p_{2} / \mu_{2}\right)$ is common when fitting a mixture of 2 exponentials to only the first two moments $E\{B\}$ and $\operatorname{Var}\{B\}$. Define $c^{2}=\operatorname{Var}\{B\} / E\{B\}^{2}$; that is, $c^{2}$ is the squared coefficient of variation (SCV) of the claim size distribution. Then the parameters $p_{i}, \mu_{i}, i=1,2$, are found from the following equations:

$$
\begin{gathered}
R=\sqrt{\left(c^{2}-1\right) /\left(c^{2}+1\right)}, \\
p_{1}, p_{2}=(1 \pm R) / 2 \\
\mu_{i}=2 p_{i} / E\{B\}
\end{gathered}
$$

Figures 7 and 8 consider the case where there is no initial reserve and $\theta=100 \%$. The SCV takes on values of 1.0 (corresponding to the ordinary exponential), 4.0 and 9.0. The figures show that around the 40th claim, the probability of being ruined on a given claim is almost 100 times more likely when SCV $=9.0$ than with the ordinary exponential. In the short-run, however, there is more chance of being ruined by a less variable claim size distribution. This seemingly contradictory result can be understood according to the following reasoning: when $\mathrm{SCV}=9$, we get $R=0.905, p_{1}=.952$ and $p_{2}=.048$. Thus, for every large claim in the long-run, there are roughly 20 smaller ones, but the rare large-sized claim is roughly 20 times larger on average. Therefore, in the very short-run, there is little chance of ruin, but eventually, the larger claims start to occur, and their impact is so much greater.

Figure 9 compares the ruin probabilities for exponential and Erlang-2 claim sizes for two values of IRR, and assuming a relative security loading of $20 \%$. As the figure demonstrates, there is substantially less likelihood of ruin for Erlang-2 claims. This is due to the reduced variability of the claim sizes. (The Erlang-2 case has a $c^{2}$ equal to half that of the exponential.) 


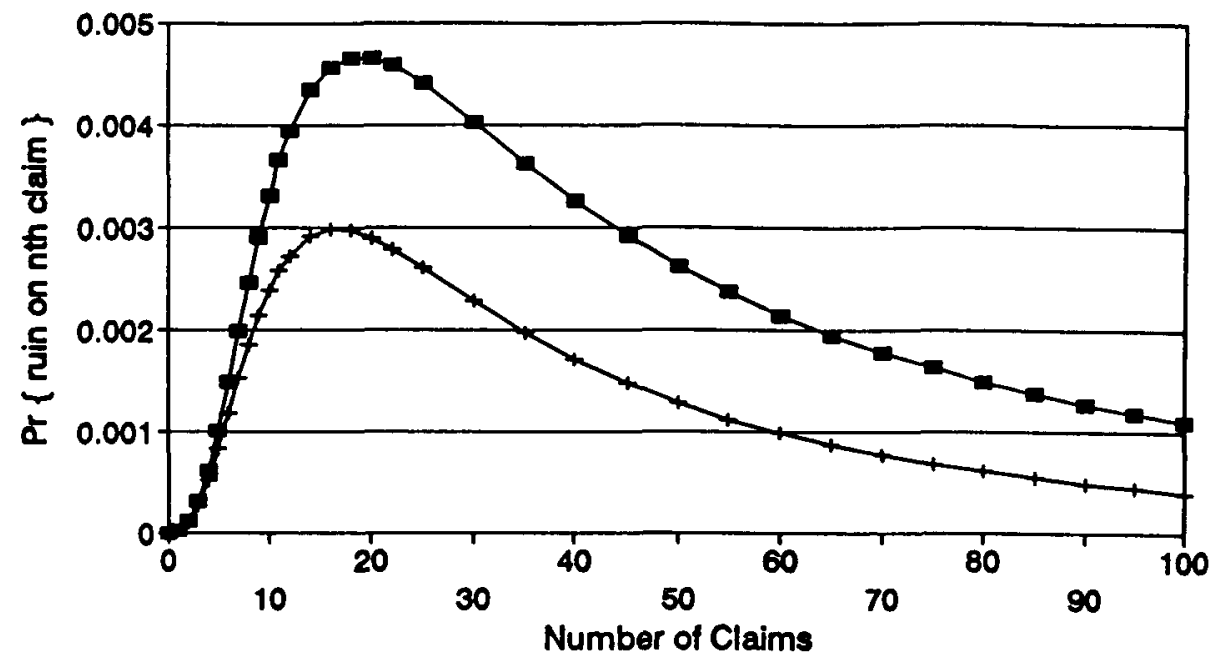

\section{$\rightarrow$ theta $=10 \%+$ theta $=20 \%$}

FIGURE 3. Effect of security loading (initial reserve ratio $=10$ ).

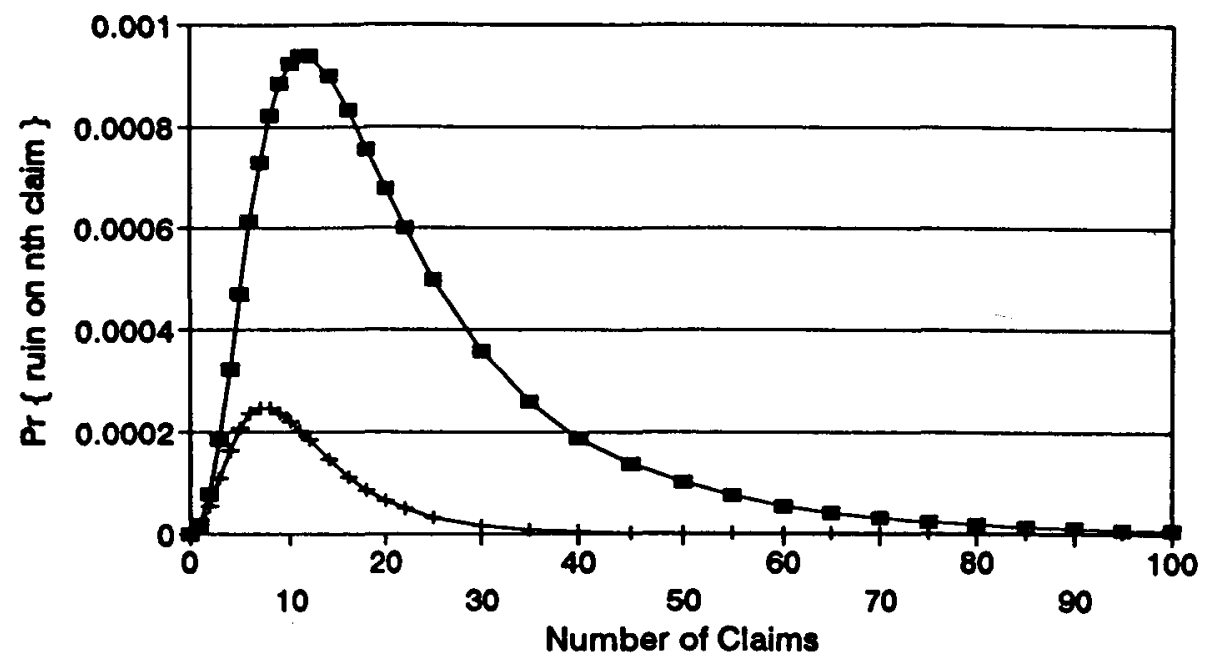

\section{$\rightarrow$ theta $=50 \%+$ theta $=100 \%$}

FIGURE 4. Effect of security loading (initial reserve ratio $=10$ ). 


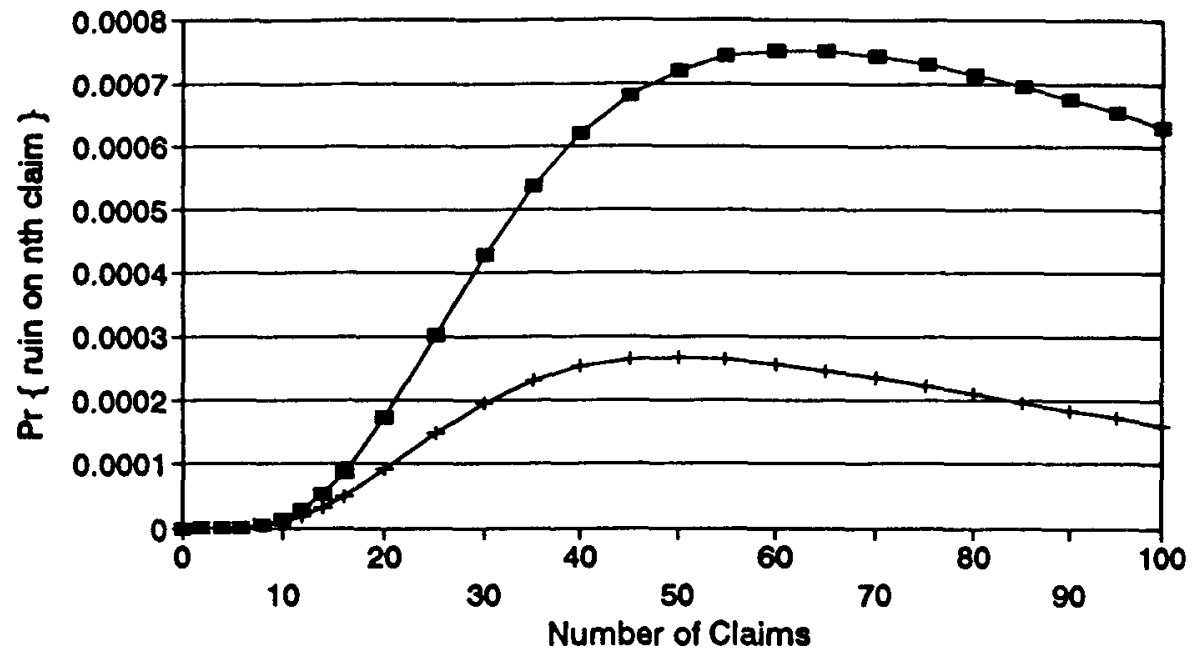

\section{$\rightarrow$ theta $=10 \%$ - theta $=20 \%$}

FIGURE 5. Effect of security loading (initial reserve ratio $=20$ ).

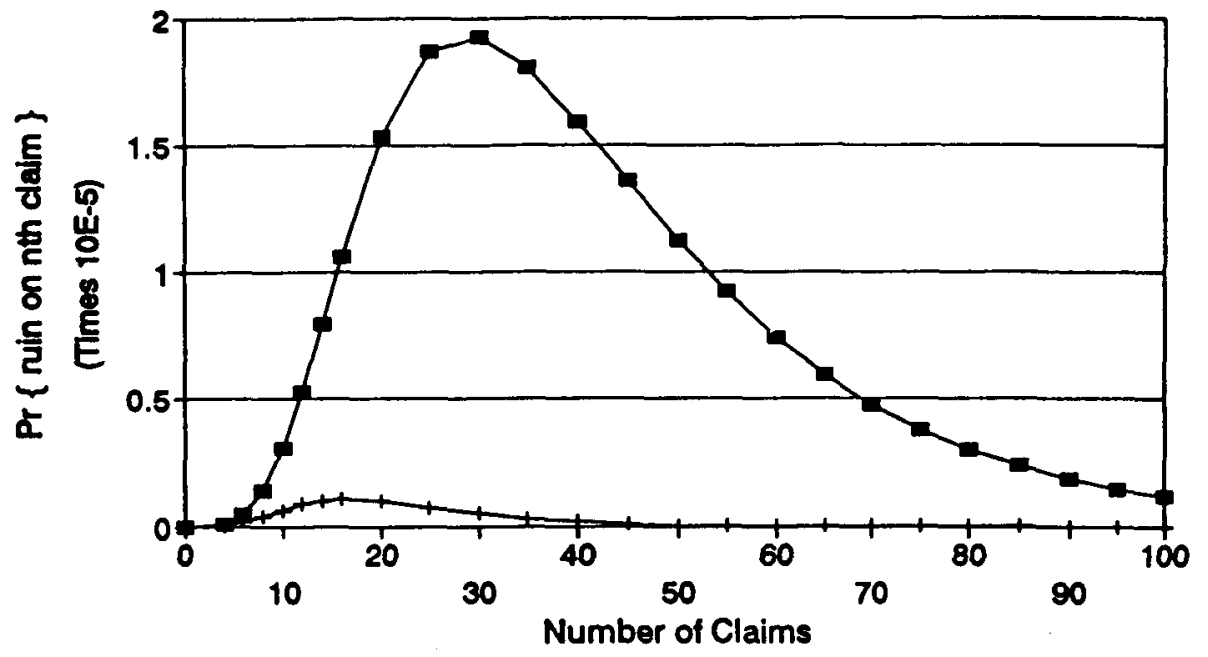

$\rightarrow$ theta $=50 \% \rightarrow$ theta $=100 \%$

FIGURE 6. Effect of security loading (initial reserve ratio $=20$ ). 


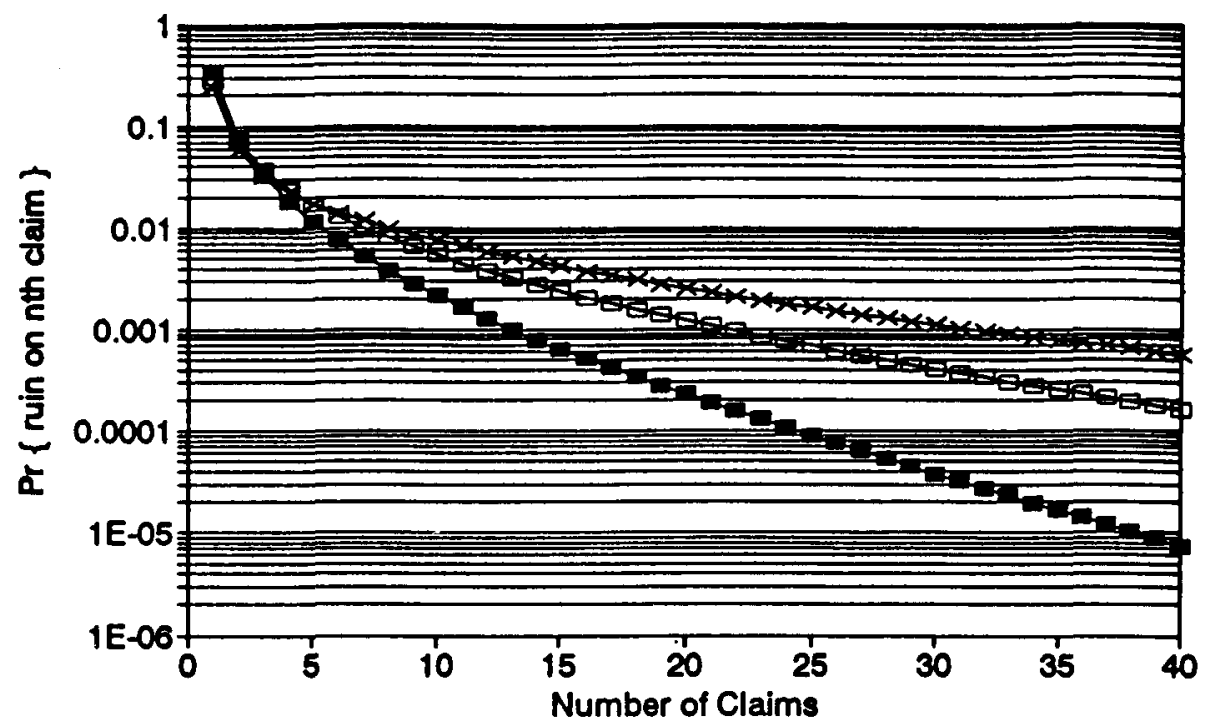

$$
\because-\mathrm{SCV}=1.00 \multimap \mathrm{SCV}=4.00 \multimap \mathrm{SCV}=9.00
$$

Figure 7. Effect of claim size variability.

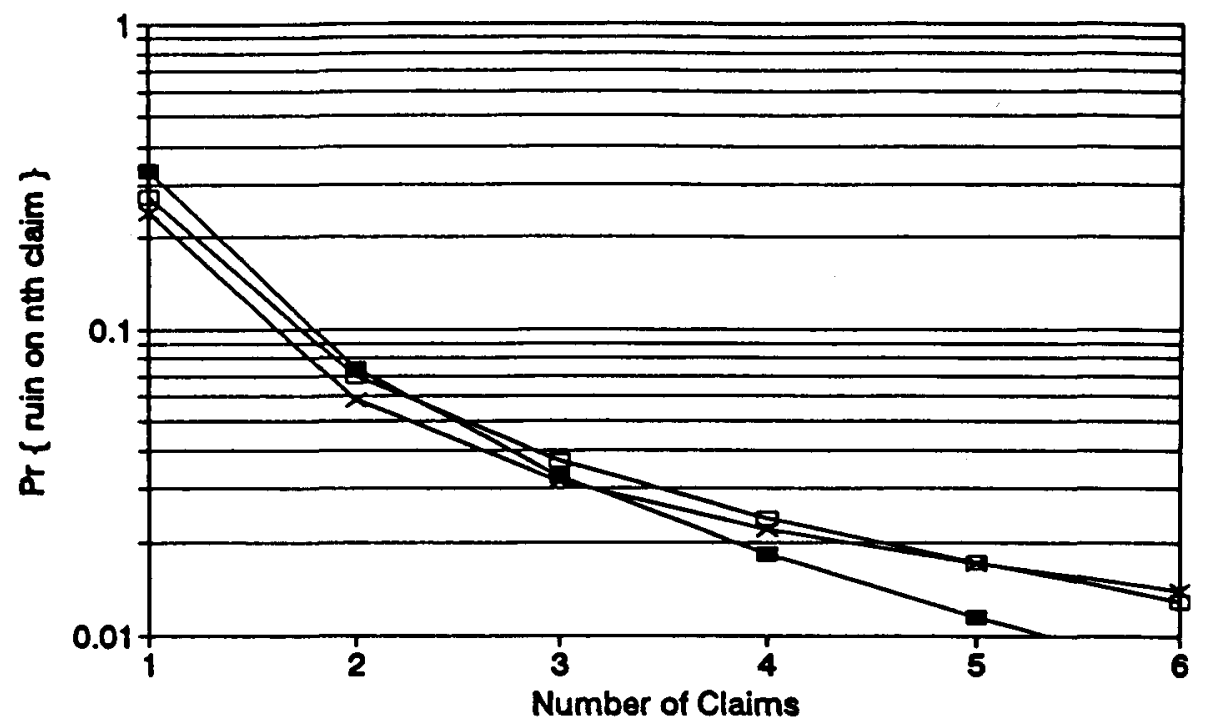

$S C V=1.00 \multimap-S C V=4.00 \rightarrow-S C V=9.00$

FIGURE 8 . Effect of claim size variability. 


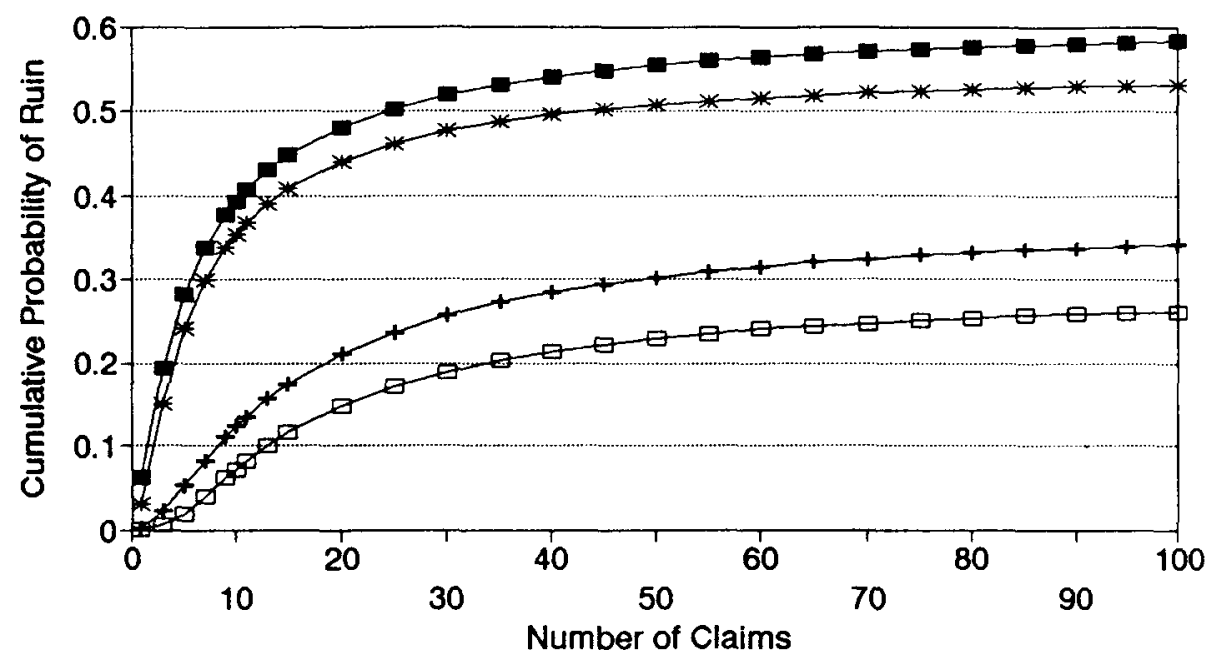

$\rightarrow-$ Exp./IRR $=2+$ Exp./IRR $=5 *$ Erl./IRR $=2$ - Erl./lRR=5

FiguRE 9. Effect of claim size distribution (security loading $=20 \%$ ).

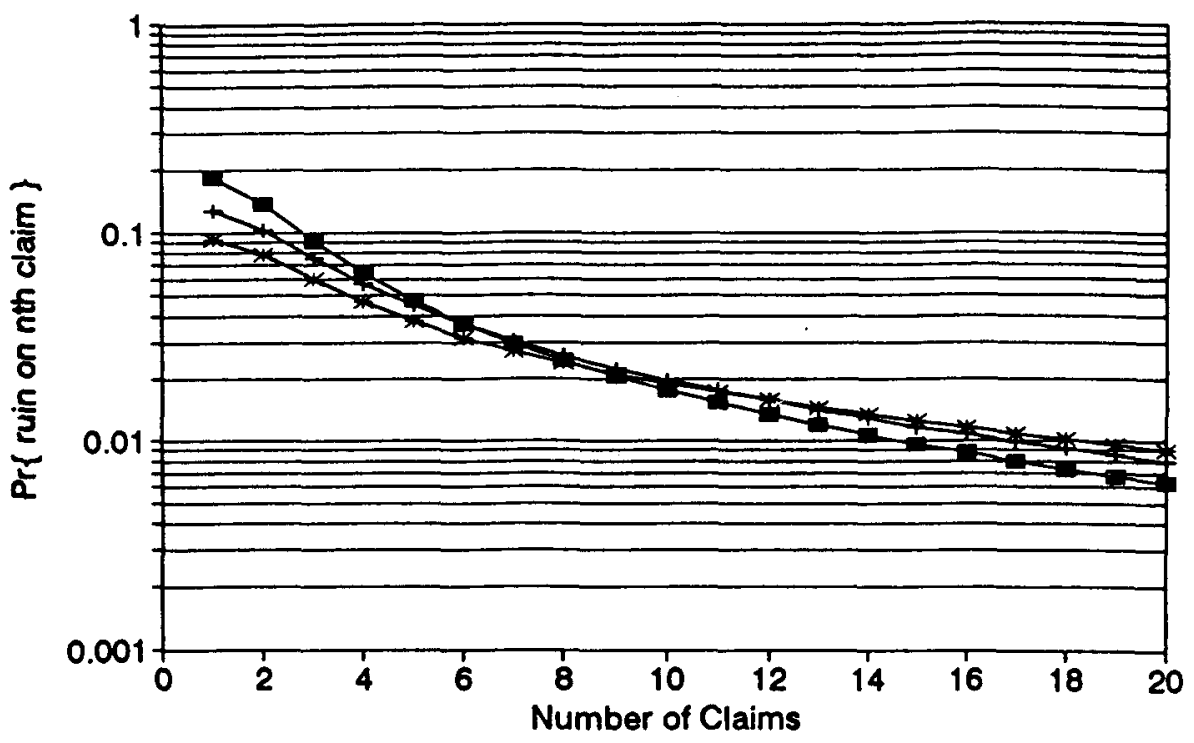

\section{$S C V=1.00+S C V=4.00 \rightarrow-S C V=9.00$}

FIGURE 10. Effect of claim size variability. 
Figure 10 provides a comparison of the ruin probabilities for the same three claim sizes distributions as Figures 7 and 8, assuming a non-zero initial reserve. The IRR equals 1 in this case, and the relative security loading is $0 \%$. Although ruin is therefore certain, we chose this example to demonstrate the ruin behaviour over finite time. Again, we see that in the short run, the exponential case has the highest likelihood of ruin, but in the long run the situation is reversed.

\section{Conclusions and Future Work}

The current paper has presented recursive methods for determining the probability of ruin at claim instants. Among the advantages of this approach are the fact that it is exact, and that it reveals where in the future that the relative vulnerability to the company lies. We hope to extend this method to include non-Poisson claims processes in further work.

\section{ACKNOWLEDGEMENTS}

We would like to thank our joint graduate student Chit Nam Chua for programming the algorithms and preparing the graphs. We would also like to thank the referees for their helpful comments. This work has been financially supported by Canada's Natural Sciences and Engineering Research Council operating grant number OGP0041187.

\section{REFERENCES}

Arfwedson, G. (1950) Some problems in the collective theory of risk. Skand. Aktuartidskr. 1-38.

Asmussen, S. and Bladt, M. (1992) Phase-type distributions and risk processes with state-dependent premiums. Technical Report R92-2022, The University of Aalborg.

Asmussen, S. and Rolski, T. (1991) Computational methods in risk theory: a matrix-algorithmic approach. Technical Report R91-13, The University of Aalborg.

BEEKMAN, J.A. (1966) Research on the collective risk stochastic process. Skand. Aktuartidskr. 65-77.

Bowers, N.L., Gerber, H.V., Hickman, J.C., Jones, D.A. and Nesbitt, C.J. (1986) Actuarial Mathematics. Society of Actuaries, Itasca, Illinois.

Cramér, M. (1955) Collective Risk Theory. Jubilee Volume of Försäkringsaktiebogalet Skandia.

DE Vyldek, F. and GoOvaERTS, M.J. (1988) Recursive calculations of finite-time ruin probabilities. Insurance: Mathematics and Economics 7, 1-8.

DiCKSON, D.C.M. and WATERS, H.R. (1991) Recursive calculation of survival probabilities. ASTIN Bulletin 21, 199-221.

DiCKSON, D.C.M. and WATERS, H.R. (1992) The probability of severity of ruin in finite and infinite time. ASTIN Bulletin 22, 177-190.

Dufresne, F. and Gerber, H.U. (1988) The probability and severity of ruin for combinations of exponential claim amount distributions and their Translations. Insurance: Mathematics and Economics 7, 75-80.

GARRIDO, J. (1988) Diffusion premiums for claim severities subject to inflation. Insurance: Mathematics and Economics 7, 123-129.

Gerber, H.U. (1979) An Introduction to Mathematical Risk Theory. Monograph No. 8, S.S. Huebuer Foundation, Distributed by R. Irwin, Homewood, IL.

Gerber, H.U., Goovaerts, M.J. and KaAs, R. (1987) On the probability and severity of ruin. ASTIN Bulletin 17, 151-163.

JANSSEN, J. (1980) Some transient results on the $M / S M / 1$ special semi-Markov model in risk and queueing theories. ASTIN Bulletin 11, 41-51.

JANSSEN, J. (1982) On the interaction between risk and queueing theories. Blätter 15, 383-395.

JanSSEN, J. and Delfosse, P. (1982) Some numerical aspects in transient risk theory. ASTIN Bulletin 13, 99-113. 
Neuts, M.F. (1981) Matrix-geometric Solutions in Stochastic Models. John Hopkins University Press, Baltimore.

PANJER, H.H. (1981) Recursive evaluation of a family of compound distributions. ASTIN Bulletin 12, 22-26.

Prabhu, N.U. (1961) On the ruin problem of collective risk theory. Annals of Math. Statistics 32, 757-764.

Prabhu, N.U. (1965) Queues and Inventories, John Wiley \& Sons, New York.

SHIU, E.S.W. (1988) Calculation of the probability of eventual ruin by Beekman's convolution series. Insurance: Mathematics and Economics 7, 41-47.

TAKÁcs, L. (1967) Combinatorial Methods in the Theory of Stochastic Processes, John Wiley \& Sons, New York.

TAYLOR, G.C. (1978) Representation and explicit calculation of finite time ruin probabilities. Scan. Actuarial Journal 78 (1), 1-18.

TAYLOR, G.C. (1985) A heuristic review of some ruin theory results. AS7IN Bulletin 15, 73-88.

THORIN, O. (1968) An identity in the collective risk theory with some applications. Skand. Aktuartidskr. 26-44.

WILLmot, G. (1990) A queueing theoretic approach to the analysis of the claims payment process. Transactions of the Society of Actuaries, XLII, 447-497. 